\title{
Pentraxin-3 and the pathogenesis of preeclampsia
}

\author{
Fabio Angeli ${ }^{1,2} \cdot$ Enrica Angeli $^{3} \cdot$ Monica Trapasso $^{4} \cdot$ Paolo Verdecchia $^{4}$
}

Received: 31 March 2020 / Revised: 2 April 2020 / Accepted: 2 April 2020 / Published online: 14 May 2020

(c) The Japanese Society of Hypertension 2020

Hypertensive disorders of pregnancy are a heterogeneous group of high blood pressure (BP) disorders that include chronic hypertension, gestational hypertension, preeclampsia-eclampsia, and other hypertensive effects (whitecoat hypertension, masked hypertension, and transient hypertension) [1,2]. More simply, hypertension in pregnancy may be chronic (predating pregnancy or diagnosed before 20 weeks of pregnancy) or occur de novo (either preeclampsia or gestational hypertension) [1].

Among de novo forms of hypertension, preeclampsia is a multisystem progressive disorder occurring in the last half of pregnancy or postpartum that is characterized by the new onset of hypertension associated with proteinuria or significant target organ dysfunction [1].

Eclampsia (the occurrence of new onset, generalized, tonic-clonic seizures, or coma) and hemolysis, elevated liver enzymes, and low platelet count (HELLP) syndrome are manifestations of preeclampsia [1].

Of note, gestational hypertension may be considered a provisional diagnosis for hypertensive pregnant women who do not meet the criteria for preeclampsia or chronic hypertension. Thus, during pregnancy, the diagnosis may change to preeclampsia, chronic hypertension, or transient hypertension.

Hypertensive disorders of pregnancy complicate $~ 6-11 \%$ of all pregnancies and are associated with adverse maternal

$\triangle$ Fabio Angeli

angeli.internet@gmail.com

1 Department of Medicine and Surgery, University of Insubria, Varese, Italy

2 Department of Medicine and Cardiopulmonary Rehabilitation, Maugeri Care and Research Institutes, IRCCS Tradate, Varese, Italy

3 Department of Obstetrics and Gynecology, San Giovanni Battista Hospital, Foligno, Italy

4 Fondazione Umbra Cuore e Ipertensione-ONLUS and Division of Cardiology, Hospital S. Maria della Misericordia, Perugia, Italy and fetal outcomes, particularly when high BP is due to preeclampsia [1]. Moreover, preeclampsia complicates $4.6 \%$ of all pregnancies each year, resulting in 50,000-60,000 deaths annually worldwide [3].

Despite decades of research, preeclampsia remains a complex medical disorder to fully understand. Indeed, preeclampsia is a multisystemic syndrome involving many genetic and environmental factors in its pathogenesis and pathophysiology.

In the last few years, the role of several markers has been tested to elucidate the pathophysiology of preeclampsia; numerous biochemical markers have been identified, including plasminogen activator inhibitor, soluble fms-like tyrosine kinase, placental growth factor, von Willebrand factor, leptin, C-reactive protein, serum uric acid, urinary proteomics, inherited thrombophilia factor $\mathrm{V}$ Leiden mutation, protein $\mathrm{C}$ or S deficiency, antithrombin III deficiency, antiphospholipid antibodies, $\alpha$-fetoprotein, human chorionic gonadotrophin, and plasma tumor necrosis factor- $\alpha$ [4]. Of note, experimental models developed to better elucidate the mechanisms involved in the development of placental and maternal vascular dysfunction in preeclamptic women strongly support the pivotal role of oxidative stress and inflammation.

In this context, the results of the Genetics and PreEclampsia (GenPE) study [5] published in the current issue of the journal offer the chance to test the biological plausibility of the role of pentraxin-3 as a novel inflammatory marker in the pathophysiology of this disorder.

Briefly, the GenPE study is a multicenter and case-control study that recruited pregnant women at the time of delivery in eight Colombian cities. Overall, 1024 pregnant women (461 controls, 368 women with preeclampsia, and 195 with HELLP syndrome) were included in the analysis. After adjustment for potential confounders (including age, ethnicity, socioeconomic status, smoking status, body mass index, gestational age, and multiple pregnancy), for each unit increase $(1 \mathrm{ng} / \mathrm{ml})$ of pentraxin-3 levels, the risk of preeclampsia and HELLP syndrome increased by $6 \%$ (odds ratio [OR]: $1.06,95 \%$ confidence 


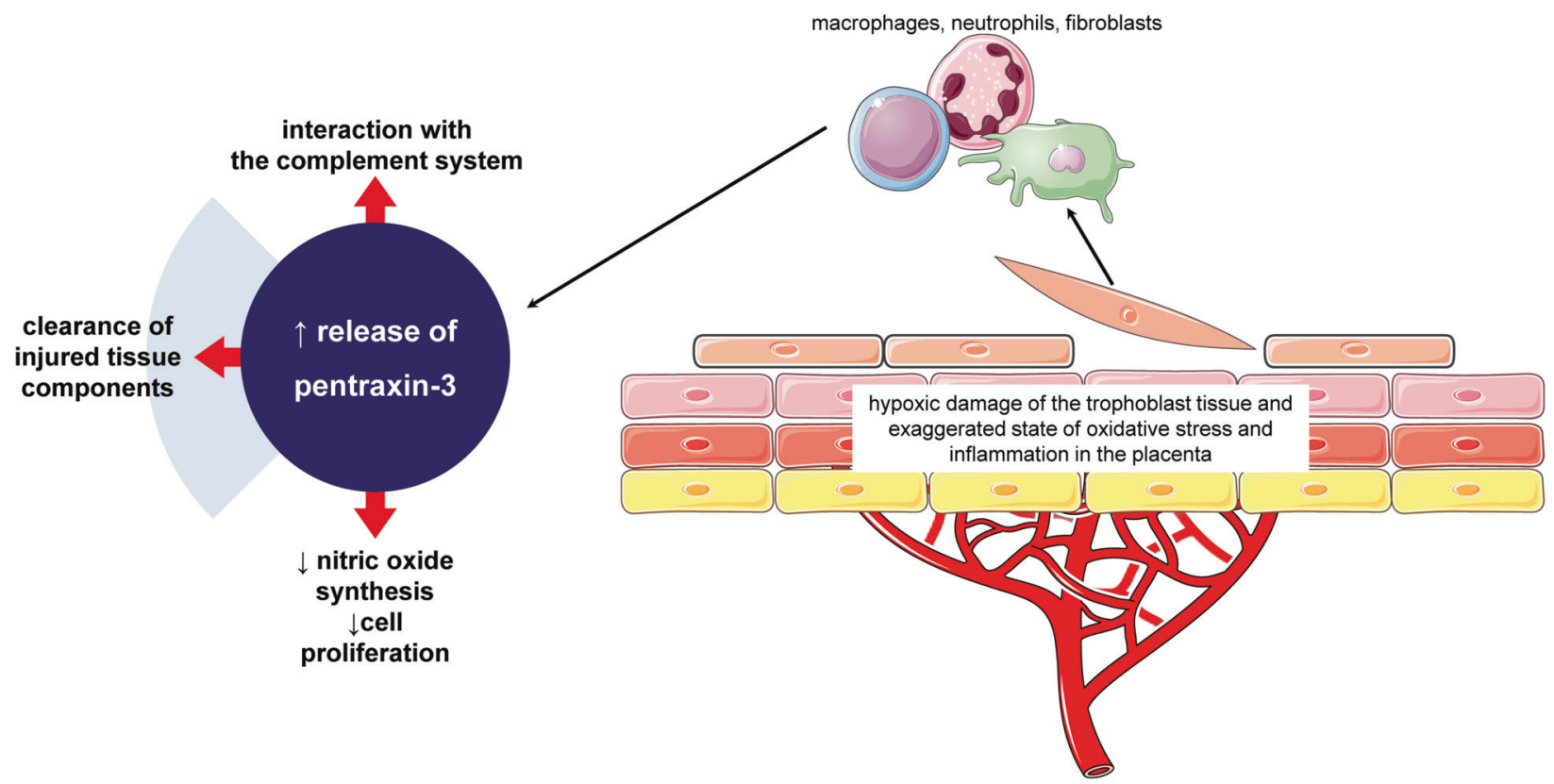

Fig. 1 Effects of pentraxin-3 (left panel) and mechanisms involved in the pathogenesis of preeclampsia (right panel). See text for details

interval $[\mathrm{CI}]: 1.03-1.10, p<0.001)$. A similar relationship was found for HELLP syndrome (OR: 1.13, 95\% CI: $1.08-1.18, p<0.001$ ).

These findings suggest the potential involvement of pentraxin-3 in the pathogenesis of severe forms of hypertensive disease in pregnancy. Nonetheless, knowledge on the functions of pentraxin- 3 and inflammatory adaptations in pregnancy is required to correctly interpret the results.

Pentraxin-3 regulates inflammation, activating and interacting with multiple components of the complement system (classical pathway), and coordinates spatially and temporally targeted clearance of injured tissue components [6].

Although mainly expressed by vascular endothelium and smooth muscle cells, pentraxin-3 is also synthesized by myeloid dendritic cells, macrophages, fibroblasts, adipocytes, synovial cells, and chondrocytes [6]. Of note, within endothelial cells, pentraxin-3 decreases nitric oxide synthesis, inhibits cell proliferation, and alters their function [6] (Fig. 1).

As mentioned above, maternal inflammation seems to be a key factor in the etiology of preeclampsia. More specifically, the fetal trophoblast is regarded as an alloantigen, and the mother reacts to this and mounts a low-grade systemic inflammatory response. To date, it is believed that in preeclamptic compared with normal pregnancies, there is an exaggerated inflammatory response and endothelial dysfunction, resulting in an increased release of inflammatory markers in the circulation (Fig. 1). Moreover, the failure to establish adequate uteroplacental blood flow in preeclamptic women can result in hypoxic damage of the trophoblast tissue, promoting an exaggerated state of oxidative stress and inflammation in the placenta [7].

In this context, the analysis by Colmenares-Mejia et al. [5] confirms the role of inflammation in the pathogenesis of preeclampsia. Specifically, the results suggest that interactions between pentraxin- 3 and the complement system are potentially also involved in conditions such as preeclampsia, where tissue damage and necrosis often occur [7] (Fig. 1). Nonetheless, and as stated by the authors [5], the usefulness of pentraxin-3 during pregnancy to stratify the risk for the development of hypertensive disease remains unproven.

In conclusion, epidemiological evidence supporting the worse prognosis associated with hypertensive disorders of pregnancy provides a strong basis for the development of risk prediction models to identify women whose gestations may be considered at high risk [8]. The identification of pregnant women requiring closer surveillance and preventive treatment is the real challenge in this setting.

Unfortunately, the mechanisms involved in the pathogenesis of hypertensive disorders of pregnancy are complex and still largely unknown. In the last few years, multiple potential biomarkers (including pentraxin-3) have been identified [4]. Nonetheless, further studies are still required to provide a better understanding of the key processes in the development of hypertension during pregnancy, and current evidence does not support the use of a single biomarker to predict severe forms of hypertension in pregnancy.

In our opinion, only a combination model including clinical, laboratory, and instrumental examinations (uterine artery Doppler assessment and electrocardiography) may 
become a robust predictive model for hypertensive disorders of pregnancy [8-10].

\section{Compliance with ethical standards}

Conflict of interest The authors declare that they have no conflict of interest.

Publisher's note Springer Nature remains neutral with regard to jurisdictional claims in published maps and institutional affiliations.

\section{References}

1. Brown MA, Magee LA, Kenny LC, Karumanchi SA, McCarthy FP, Saito S, et al. Hypertensive disorders of pregnancy: ISSHP classification, diagnosis, and management recommendations for international practice. Hypertension. 2018;72:24-43.

2. Poon LC, Shennan A, Hyett JA, Kapur A, Hadar E, Divakar H, et al. The International Federation of Gynecology and Obstetrics (FIGO) initiative on pre-eclampsia: a pragmatic guide for firsttrimester screening and prevention. Int $\mathrm{J}$ Gynaecol Obstet. 2019;145:1-33.
3. Abalos E, Cuesta C, Grosso AL, Chou D, Say L. Global and regional estimates of preeclampsia and eclampsia: a systematic review. Eur J Obstet Gynecol Reprod Biol. 2013;170:1-7.

4. Angeli F, Angeli E, Reboldi G, Verdecchia P. Hypertensive disorders during pregnancy: clinical applicability of risk prediction models. J Hypertens. 2011;29:2320-3.

5. Colmenares-Mejía CC, Quintero-Lesmes DC, Bautista-Niño PK, Guio Mahecha E, Beltrán Avendaño M, Díaz Martínez LA, et al. Pentraxin-3 is a candidate biomarker on the spectrum of severity from pre-eclampsia to HELLP syndrome: GenPE study. Hypertens Res. 2020 https://doi.org/10.1038/s41440-020-0434-0.

6. Haapasalo K, Meri S. Regulation of the complement system by pentraxins. Front Immunol. 2019;10:1750.

7. Myatt L. Role of placenta in preeclampsia. Endocrine. 2002; 19:103-11.

8. Angeli F, Angeli E, Verdecchia P. Novel electrocardiographic patterns for the prediction of hypertensive disorders of pregnancyfrom pathophysiology to practical implications. Int J Mol Sci. 2015;16:18454-73.

9. Angeli F, Angeli E, Verdecchia P. Electrocardiographic changes in hypertensive disorders of pregnancy. Hypertens Res. 2014;37: 973-5.

10. Angeli E, Verdecchia P, Narducci P, Angeli F. Additive value of standard ECG for the risk prediction of hypertensive disorders during pregnancy. Hypertens Res. 2011;34:707-13. 